\title{
A Model for the CD4 Cell Counts in an HIV/AIDS Patient and its Application in Treatment Interventions
}

\author{
${ }^{1}$ Richard O. Simwa and ${ }^{2}$ Joseph Y.T. Mugisha \\ ${ }^{1}$ Department of Mathematics, University of Nairobi, Box 30197, Nairobi, Kenya \\ ${ }^{2}$ Department of Mathematics, Makerere University, Box 7062, Kampala, Uganda
}

\begin{abstract}
The number of CD4 white blood cells has been established as an important clinical marker of disease progression to acquired immunodeficiency syndrome (AIDS) for persons infected with human immunodeficiency virus (HIV). The number of CD4 cells per unit volume is expected to decrease with time since infection by the virus. However on introduction of treatment interventions, the process is expected to reverse with the counts increasing to return to the normal level. In this study we deduce that the count per unit volume of blood of an HIV/AIDS patient has a linear relationship with the time since infection during the short period of time immediately treatment usage begins. We show one application of the model in treatment selection strategy.
\end{abstract}

Key words: CD4 cells, cell count, treatment, interventions

\section{INTRODUCTION}

An understanding of typical course of the infection within an individual and the quantitative information about latent period, infectious period or in some cases incubation period is essential in designing effective treatment strategies. In a given population, it is considered desirable to classify individuals (further stratified by spatial locality, age or gender) for modeling purposes into the categories that are:

* Immunized (protected from the infection by maternally derived factors or through vaccination)

* Susceptible (not yet infected but may get infected if exposed to the disease)

* Infected (and in the various stages of infection)

* Permanently removed (AIDS or dead)

In order to describe the various stages of HIV infection one can make use of the corresponding differential equations showing the rates of changes in the number of individuals at the respective stages with the help of relevant parameters ${ }^{[1,2]}$. Simwa and Pokhariyal ${ }^{[3]}$ constructed a dynamical model for stagespecific HIV incidences, using two systems of ordinary differential equations that are coupled through a delay in one of the systems. Further by incorporating possible vaccine interventions in the dynamical stage specific model simulations of the HIV pandemic show the vaccine effect on the stage specific projections ${ }^{[4]}$.

Other forms of interventions include use of antiretroviral (ARV) drugs by the HIV infected patients. These drugs include AZT, among others. In this study the effect of such drugs to a patient is considered. The CD4 cells forms a group of white cells that provide immune capability to ones body. The HIV virus is known to attack and destroy these cells once an individual is infected. The depletion of the cells leads to loss of body immunity and thus AIDS. In fact the absolute number of CD4 cells has been established as a significant predicator of disease progression in the patient ${ }^{[5]}$. However application of the anti-retroviral drugs may lead to reduced rate of destruction of these cells by the virus such that the cell count per unit volume of blood of the patient starts increasing. We model the CD4 cells counts per unit volume of blood in an HIV patient as from time of treatment application, as a function of time since infection. Theories from survival and actuarial studies are adapted to analyse the distribution of the future lifetime of an arbitrary newly formed CD4 cell in an HIV infected patient. An expression for the counts per unit volume (milliliter) as a linear function of the hazard rate of the distribution of the CD4 cell lifetime on commencement of treatment is derived in the next section. From these results we deduce strategies that can be used in the analysis of treatment interventions, with the analysis based on Simwa $^{[6]}$.

The model: Let $N(t)$ be the number of CD4 cells per unit volume of blood in an individual's body at time $t$. $N(t)$ is a random variable and if

$$
\mathrm{N}=\mathrm{E}[\mathrm{N}(\mathrm{t})]
$$

is the expected value of $N(t)$, then the estimate for $N$ for a healthy person has been approximated to be 1200 per $\mathrm{mm}^{3[5]}$. For an HIV patient it has been noted that this number reduces to, say

$\mathrm{NP}(\tau)<1200$

Corresponding Author: Joseph Y.T. Mugisha, Department of Mathematics, Makerere University, Box 7062, Kampala, Uganda 
Where, $\tau$ is the duration in, say years, since the HIV infection and $0<P(\tau) \leq 1$. Thus, $0<\tau<$ (incubation period). Let $N(\tau, t)$ denote the number of CD4 cells per unit volume at time $t$ in the body of an HIV patient infected $\tau$ years ago. $N(\tau, t)$ is also a random variable and we let its expected value be denoted by $N_{\tau}$, that is,

$$
\mathrm{N}_{\tau}=\mathrm{E}[\mathrm{N}(\tau, \mathrm{t})]
$$

Thus,

$$
\mathrm{N}_{\tau}=\mathrm{NP}(\tau)
$$

We next derive the expression of $P(\tau)$ in terms of some mean parameters and the exact expression of $N_{\tau}$, (equations 2.26, 2.27 and 2.28).

A model for $P(\tau)$ : We show that a possible model for $P(\tau)$ is given by

$$
\mathrm{P}(\tau)=\frac{\mu(\tau)}{\mu}
$$

Where, $\mu$ is the expected lifetime of a CD4 cell in a healthy person and $\mu(\tau)$ is the expected lifetime of a CD4 cell in an HIV patient.

Let $T_{\tau}(t)$ denote the future lifetime of a newly formed CD4 cell at time $\mathrm{t}$ in an individual infected $\tau$ years ago and $T(t)$ denote the future lifetime of a newly formed CD4 cell in a healthy individual at time $t$. Both the quantities $T_{\tau}(t)$ and $T(t)$ for $t \geq 0, \tau \geq 0$ each form a stochastic process with means to be denoted by $\mu(t, \tau)$ and $\mu(t)$ respectively. Let $P(t, \tau)$ be the proportion at time $t$ of the $N$ CD4 cells per unit volume that are observed in an HIV patient infected $\tau$ years ago. It follows that

$\mathrm{P}(\tau)=\int_{\mathrm{t}=0}^{\infty} \omega(\mathrm{t}, \tau) \mathrm{P}(\mathrm{t}, \tau) \mathrm{dt}$

Where, the function $\omega(t, \tau)$ gives the distribution at time t of the CD4 cells in a patient infected $\tau$ years ago such that

$$
\int_{0}^{\infty} \omega(\mathrm{t}, \tau) \mathrm{dt}=1
$$

Proposition 1: $\mathrm{P}(\mathrm{t}, \tau)=\frac{\mathrm{E}\left[\mathrm{T}_{\tau}(\mathrm{t})\right]}{\mathrm{E}[\mathrm{T}(\mathrm{t})]}=\frac{\mu(\tau, \mathrm{t})}{\mu(\mathrm{t})}$
Proof: A CD4 cell in an HIV patient is exposed to extra risk of dying due to the attack by the HIV viruses and hence the average lifetime of the cells is less than the case before the infection. Hence we may adopt the theories on Exposed-to-Risk to estimate $P(t, \tau)$. It is noted that $P(t, \tau)$ can also be taken as the ratio of the expected total exposure time (expected total lifetime) of CD4 cells to the risk of dying at time $t$ with and without infection, of a given constant number of these cells, say $k(t)$ cells.

For a healthy HIV non infected individual at time $t$,

Total exposure time $=\sum_{j=1}^{k(t)} T(t, j)$

where $T(t, j)$ is the lifetime of the $j^{t h} \mathrm{CD} 4$ cell $(j=1,2, \ldots, k(t))$. On the other hand, for an HIV infected patient (infected $\tau$ years ago),

total exposure time $=\sum_{\mathrm{j}=1}^{\mathrm{k}(\mathrm{t})} \mathrm{T}_{\tau}(\mathrm{t}, \mathrm{j})$

where $T_{\tau}(t, j)$ is the lifetime of the $j^{\text {th }} \mathrm{CD} 4$ cell $(j=$ $1,2, \ldots, k(t))$. Thus,

$$
\begin{aligned}
\mathrm{P}(\mathrm{t}, \tau) & =\frac{\lim _{\mathrm{k}(\mathrm{t}) \rightarrow \infty} \mathrm{E}\left[\sum_{\mathrm{j}=1}^{\mathrm{k}(\mathrm{t})} \mathrm{T}_{\tau}(\mathrm{t}, \mathrm{j})\right]}{\lim _{\mathrm{k}(\mathrm{t}) \rightarrow \infty} \mathrm{E}\left[\sum_{\mathrm{j}=1}^{\mathrm{k}(\mathrm{t})} \mathrm{T}(\mathrm{t}, \mathrm{j})\right]} \\
= & \frac{\lim _{\mathrm{k}(\mathrm{t}) \rightarrow \infty} \mathrm{k}(\mathrm{t}) \times \mathrm{E}\left[\mathrm{T}_{\tau}(\mathrm{t})\right]}{\lim _{\mathrm{k}(\mathrm{t}) \rightarrow \infty} \mathrm{k}(\mathrm{t}) \times \mathrm{E}[\mathrm{T}(\mathrm{t})]}=\frac{\mu(\tau, \mathrm{t})}{\mu(\mathrm{t})}
\end{aligned}
$$

Note that we have assumed that $\mathrm{T}_{\tau}(\mathrm{t}, \mathrm{j}) ; \quad \mathrm{j}=1,2, \ldots, \mathrm{k}(\mathrm{t})$ form a random sample from the distribution of the random variable $T_{\tau}(t)$ while $T(t, j)$; $j=1,2, \ldots, k(t)$ form a random sample from the distribution of the random variable $T(t)$ and that time $t$ may be the calendar time or age of an individual. Thus the prove to the proposition.

Now, assume $\mu(t)=\mu$; that the mean lifetime of a CD4 cell in a healthy individual is on average independent of time t, the age of the person (or calendar year) as expected. Also let $\mu(\tau, t)=\mu(\tau)$; that in an HIV infected person the mean lifetime of a CD4 cell depends only on the time since infection. Then

$$
\begin{aligned}
\mathrm{P}(\tau) & =\int_{0}^{\infty} \omega(\mathrm{t}, \tau) \mathrm{P}(\mathrm{t}, \tau) \mathrm{dt}=\int_{0}^{\infty} \omega(\mathrm{t}, \tau) \frac{\mu(\tau, \mathrm{t})}{\mu(\mathrm{t})} \mathrm{dt} \\
& =\frac{\mu(\mathrm{t})}{\mu} \int_{0}^{\infty} \omega(\mathrm{t}, \tau) \mathrm{dt}=\frac{\mu(\tau)}{\mu}
\end{aligned}
$$

This completes the prove to the main result on $P(\tau)$. 
The result below gives $\mu(\tau)$ as a function of the hazard function for the distribution of the lifetime of a CD4 cell.

Proposition 2: $\mu(\tau) \approx \omega\left(1-\frac{\omega \lambda(\tau)}{2}\right)$

where $\omega$ is the maximum (limiting) age or lifespan and $\lambda(\tau)$ is the hazard function of the distribution of the future lifetime of an arbitrary newly formed CD4 cell in an HIV patient who was infected $\tau$ years ago.

Proof: Let $\mathrm{s}(\tau, \mathrm{t})=$ prob. $\left(\mathrm{T}_{\tau}>\mathrm{t}\right)$

And $\lambda(\tau, \mathrm{t})=\lim _{\mathrm{dt} \rightarrow 0} \frac{\operatorname{prob} .\left(\mathrm{t}<\mathrm{T}_{\tau}<\mathrm{t}+\mathrm{dt}\right)}{\operatorname{prob} .\left(\mathrm{T}_{\tau}>\mathrm{t}\right)}$

Then $s(\tau, t)$ and $\lambda(\tau, t)$ are the survival and the hazard function for the distribution of the lifetime of a CD4 cell in an infected patient who was infected $\tau$ years ago respectively.

From theories on survival analysis ${ }^{[7]}$, note that

$\mathrm{s}(\tau, \mathrm{t})=\exp -\int_{0}^{\mathrm{t}} \lambda(\tau, \mathrm{t}) \mathrm{dt}$

Assume

$\lambda(\tau, \mathrm{t})=\lambda(\tau)$

that the hazard function is independent of the 'age' of the CD4 cell (as sometimes assumed in demography $)^{[8,9]}$, but depends on time since HIV infection of the individual. Then

$\mathrm{s}(\tau, \mathrm{t})=\exp -\int_{0}^{\mathrm{t}} \lambda(\tau) \mathrm{dt}=\exp -\lambda(\tau) \mathrm{t}$

The Taylor series expansion of $s(\tau, t)$ leads to the approximation;

$\mathrm{s}(\tau, \mathrm{t}) \approx 1-\lambda(\tau) \mathrm{t}$

Note that

$\mu(\mathrm{t})=\int_{0}^{\omega} \mathrm{s}(\tau, \mathrm{t}) \mathrm{dt}$

where, $\omega$ is the maximum (limiting) 'age' of a CD4 cell $^{[7]}$. Thus, $\mu(\tau)=\int_{0}^{\omega} \mathrm{s}(\tau, \mathrm{t}) \mathrm{dt} \approx \int_{0}^{\omega} 1-\mathrm{t} \lambda(\tau) \mathrm{dt}=\omega\left(1-\frac{\omega \lambda(\tau)}{2}\right)$

Hence, the proof of the proposition.

Therefore, the average number of CD4 cells per $\mathrm{mm}^{3}$ in an HIV patient infected $\tau$ years ago

$$
\mathrm{N}_{\tau}=\mathrm{NP}(\tau) \approx \frac{\mathrm{N}_{\omega}}{\mu}\left(1-\frac{\omega \lambda(\tau)}{2}\right)
$$

A model for $\lambda(\tau)$ : The hazard rate $\lambda(\tau)$ is constant for a given $\tau$, it is assumed not to depend on the 'age' of the CD4 cell. However due to increased depletion of the cells by the HIV viruses we expect $\lambda(\tau)$ to increase as $\tau \rightarrow \infty$. But from above we note that

$\frac{\mathrm{dN}(\tau)}{\mathrm{d} \tau}=-\frac{\omega^{2}}{2} \frac{\mathrm{d} \lambda(\tau)}{\mathrm{d} \tau}$

such that as $\lambda(\tau)$ increases $N(\tau)$ decreases for $\tau \geq 0$. Let $X$ denote the incubation period of AIDS with $h(x)$ as the hazard function of the distribution of the incubation period. Kiuchi et al. ${ }^{[5]}$ note that in the absence of treatment as $x$ increases $h(x)$ increases while $N(x)$ decreases. For a treatment that has been developed to improve the immune system of an HIV patient we expect that once the patient takes up the treatment, say $\tau^{*}$ time units since infection, $h(\tau)$ decreases while $N(\tau)$ increases initially for $\tau \geq \tau^{*}$. We assume the treatment act by at least reducing the rate of destruction of the CD4 cells by the invading HIV viruses. Thus, in either case

$\frac{\mathrm{dN}(\tau)}{\mathrm{d} \tau}=-\mathrm{k} \frac{\mathrm{dh}(\tau)}{\mathrm{d} \tau}$

where $k>0$ and $h(\tau)$ is an increasing function of $\tau$ for $\tau<\tau^{*}$ but is a decreasing function of $\tau$ for $\tau \geq \tau^{*}$. Combining these results (equation 2.23 and 2.24) we have

$\lambda(\tau)=\operatorname{ch}(\tau)+\mathrm{d}$

where, $c \geq 0$ and $d$ is some constant.

Hence,

$\mathrm{N}_{\tau}=\mathrm{NP}(\tau) \approx \frac{\mathrm{N}_{\omega}}{\mu}\left(1-\frac{\omega(\operatorname{ch}(\tau)+\mathrm{d})}{2}\right)$ 
The Weibull distribution has been assumed to approximate the distribution of $X^{[10,11]}$, such that

$$
h(\tau)=\alpha \gamma \tau^{\alpha-1} \text { for } \tau<\tau^{*}
$$

where, $\alpha$ and $\gamma$ are some parameters. When treatment is availed and taken by the HIV patient this model for $h(\tau)$ may not hold. However the function must be nonnegative and decreasing for $\tau \geq \tau^{*}$ for at least a short interval of time thereafter. A linear approximation to $h(\tau)$ for $\tau \geq \tau^{*}$ may be assumed. Thus,

$$
\mathrm{N}_{\tau} \approx \frac{\mathrm{N}_{\omega}}{\mu}\left(1-\frac{\omega(\operatorname{ch}(\tau)+\mathrm{d})}{2}\right)=\mathrm{a}+\mathrm{b} \tau, \tau \in\left(\tau^{*}, \tau^{*}+\delta \tau\right)(2
$$

where, $a$ and $b$ are some constants and $\delta \tau$ is a small change in $\tau$, say $\delta \tau<1$ year. $b$ is the slope parameter. Note that in the short time interval after $\tau^{*}$ time units since the HIV infection, $h(\tau)$ is a decreasing function so that its linear approximation has a negative slope and thus $b>0$ for any treatment form.

Model application in treatment selection: In this section we illustrate the application of the model in HIV/AIDS treatment intervention in measuring the relative effectiveness of a treatment and thus provide a possible procedure for selecting an optimal treatment form. Fig. 1 will be used to describe the procedure.

HIV infection leads to a gradual reduction in the number of CD4 cells while treatment by the ARV drugs is expected to lead to an initial abrupt increase in this number. If $N_{1}$ and $N_{2}$ are the average numbers before and after the infection at time 0 and $t_{2}\left(=\tau^{*}\right)$ years respectively since the infection, treatments type $X$ and type $Y$ may lead to the curves sketched in the figure. Treatment type $X$ requires duration of length $t_{x}-t_{2} \geq t_{y}-t_{2}$, the corresponding length with respect to treatment type $Y$, to get the CD4 cells counts back to the normal level, $N_{1}$ ("healing phase" period). Thus, the magnitude (and sign) of the slope of either curve during time interval $\left(t_{2}, t_{x}\right)$ or $\left(t_{2}, t_{y}\right)$ provides a relative measure of effectiveness of the treatments.

Therefore, the following steps could lead to classifying various types of treatment forms that may be available for the "healing phase" of an HIV infected patient under treatment.

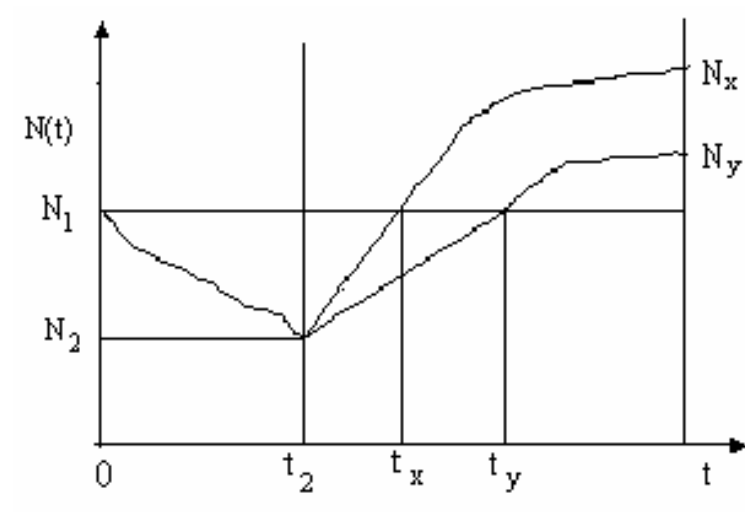

Wurber of years sirue HIV infection

Fig. 1: CD4 counts per unit volume since HIV infection

Step 1: For each patient, once treatment begins, record time of treatment and the corresponding CD4 cells count per millilitre for several treatment sessions and treatment forms. A patient is assumed to undergo one type of treatment.

Step 2: Assume linearity for a specified short time interval and determine the slopes of the curve. The approximate duration of the interval can be estimated by observing the plot of the relevant data ( $t_{x}$ and $t_{y}$ in Fig. 1).

Step 3: Test whether there is marked and significant relative treatment effects basing on the slope parameter. Then select the predominant treatment form, if any. For instance in Fig. 1, we note that the slope parameter estimates are such that $\hat{b}_{x}>\hat{b}_{y}$ and hence treatment type $X$ is better than treatment type $Y$. However standard statistical tests may be used for more rigorous analysis.

\section{ACKNOWLEDGEMENT}

The authors would like to thank NUFU for financial assistance and the anonymous referees for the helpful comments on the manuscript.

\section{REFERENCES}

1. Anderson, R.M. and R.M. May, 1995. Infectious Diseases of Humans, Dynamics and Control. Oxford Science Publications, Oxford.

2. Musekwa, S.D.H., 2001. Ph.D. Thesis. University of Zimbabwe.

3. Simwa, R.O. and G.P. Pokhariyal, 2003. A dynamical model for stage-specific HIV incidences with application to Sub-Saharan Africa. Appl. Math. Comput., 146: 93-104. 
4. Simwa, R.O., 2003b. A vaccine/treatment HIV stage-specific dynamical model. In: Proc. IBS SUSAN Conference, 7th-11th, July, Natal, South Africa.

5. Kiuchi, A.S., J.A. Hartigan, T.R. Halford, P. Rubistein and C.E. Stevens, 1995. Change points in the series of T4 counts prior to AIDS. Biometrics, 51: 236-248

6 Simwa, R.O., 2003c. A stochastic model for the CD4 cells counts in an HIV/AIDS patient and its application in treatment interventions. In: Proceedings of the 1st Pan-African Biomathematics Congress 8th-12th, December, 2003, Kampala, Uganda.

7. Klein, P.J. and M.L. Moeschberger, 1999. The Mean Residual Life Function and Median Life. In: Survival Analysis: Techniques for Censored and Truncated Data. Springer-Verlag. New York, Berlin, Delberg, pp: 32-33.
8. Abbas, A.K., A.H. Lichman and J.S. Pober, 1994. Cellular and Molecular Immunology. 2nd Edn., Philadelphia, W. B. Saunders, pp: 419-422 and 425-426.

9. May, R.M., R.M. Anderson and A.R. McLean, 1988. Possible demographic consequences of HIV/AIDS epidemic. I. Assuming HIV infections always leads to AIDS. Math. Biosci., 90: 475-505.

10. Isham, V., 1988. Mathematical Modeling of the transmission dynamics of HIV infection and AIDS: A review. J. Royal Stat. Soc., Ser. A, 151: 5-49.

11. Simwa, R.O., 2003a. On empirical modeling of HIV/AIDS pandemic with application to East Africa. African J. Sci. Technol., 4: 34-43. 